\title{
Review
}

\section{Mechanisms mediating caspase activation in cell death}

\author{
S Kumar ${ }^{\star, 1,2}$ \\ ${ }^{1}$ Hanson Centre for Cancer Research, Institute of Medical and Veterinary \\ Science, Frome Road, Adelaide, Australia; \\ 2 Department of Medicine, University of Adelaide, Adelaide, Australia \\ * Corresponding author: S Kumar, Hanson Centre for Cancer Research, IMVS, \\ PO Box 14, Rundle Mall, Adelaide, SA 5000, Australia. \\ Fax: +61-8-8222-3139; E-mail: sharad.kumar@imvs.sa.gov.au
}

Received 23.4.99; revised 17.8.99; accepted 21.9.99

Edited by D Nicholson

\begin{abstract}
The initial activation of a caspase in a caspase cascade is a crucial event that determines whether a cell will ultimately undergo cell death. Although each cell contains a number of different caspases, only a small subset may be required for apoptosis in response to a specific stimulus. It now seems that each caspase cascade has two types of caspases involved, the upstream or class I caspases, and the downstream or class II caspases. Class I caspases are characterised by long amino-terminal prodomains that carry specific proteinprotein interaction domains which mediate oligomerisation of caspases, often assisted by specific adaptor molecules. Oligomerisation appears to be sufficient for autocatalytic activation of class I caspases. Once the first caspase in the pathway has been activated, it processes downstream caspases initiating a cascade of amplifying events that lead to the apoptotic death of a cell. This article reviews our current understanding of mechanisms that mediate the activation of caspases.
\end{abstract}

Keywords: apoptosis; caspases; prodomain; oligomerisation

Abbreviations: CARD, caspase recruitment domain; $D D$, death domain; DED, death affector domain

\section{Introduction}

As reviewed on numerous previous occasions ${ }^{1-4}$ and elsewhere in this issue, caspases are the mammalian homologues of the Caenorhabditis elegans death protease CED-3. These cysteine proteases, which cleave their substrates following an aspartate residue, are normally present as inactive precursors in cells. Upon receiving an apoptotic signal, the pro-forms (zymogens) of caspases undergo proteolytic processing to generate active enzyme. The structural studies on active caspase- 1 and caspase-3 predict that the mature enzyme is a heterotetramer, composed of two heterodimers derived from two precursor molecules. ${ }^{5-8}$ In addition to the regions that give rise to two subunits, procaspases contain amino terminal prodomains of varying lengths. Based on the length of prodomain, caspases can be divided into two groups: class I, which contain a relatively long prodomain, and class II containing a short prodomain. ${ }^{9}$ From the studies during the past 2 years, it has become clear that the long prodomains in many class I caspases consist of specific protein-protein interaction domains that play a crucial role in caspase activation. These prodomains seem to serve two functions: mediate oligomerisation of the procaspase molecules and/or help recruitment of caspase precursors to specific death complexes. The oligomerisation of procaspase molecules results in caspase activation by autocatalysis, by mechanisms which are not entirely understood at present. Class II caspases which lack a long prodomain, also lack the ability to selfactivate and appear to require cleavage by activated class I caspases. For this reason, class I caspases are also referred to as initiator or upstream caspases, and class II caspases as executioner or downstream caspases. The activation of class I caspases is of fundamental importance in cell death commitment and hence substantial recent efforts have been devoted to the understanding of mechanisms that underlie caspase activation. Although it is probably premature to believe that all class I caspases are activated in a similar fashion, some concensus is emerging that caspase activation occurs by oligomerisation. This article is an attempt to bring together what is currently known about the mechanisms that mediate caspase activation.

\section{Prodomains of class I caspases}

As mentioned above, a number of caspases, both in invertebrates and in vertebrates, contain a large prodomain at their amino terminus (Table 1). Among the 14 unknown mammalian caspases, ten contain a relatively large prodomain. ${ }^{1-4,10,11}$ Two Drosophila, and four $C$. elegans caspases also carry long prodomains (Table 1). The first direct evidence for the involvement of a caspase prodomain in caspase activation came from the discovery that the activated death receptor, Fas/APO-1/CD95, can recruit caspase-8, via binding to the adaptor FADD. ${ }^{12,13}$ The interaction between Fas and FADD is mediated by death domains (DDs) present in both these proteins, whereas the interaction between FADD and caspase- 8 is mediated by death effector domains (DEDs). ${ }^{14}$ Two copies of DEDs are present in the prodomain of caspase-8, one of which interacts with the single DED in FADD molecule. ${ }^{15}$ Similarly, caspase-10 contains two DEDs and is thought to interact with FADD. ${ }^{14}$

The prodomains of several caspases contain another protein - protein interaction domain termed 'caspase recruitment domain' (CARD). ${ }^{16} \mathrm{~A}$ single copy of CARD is found in proapoptotic mammalian caspases such as caspase-2 and 
caspase-9, the $C$. elegans death protease CED- $3,{ }^{16}$ and the recently discovered Drosophila caspase DRONC. ${ }^{17}$ CARDs are also found in several other caspases, some of which, such as caspase-1 and caspase-11, primarily function in processing proinflammatory cytokines (Table 1). Similar to DEDs, CARDs seem to mediate the interaction between caspases and their respective adaptors (Table 2). For instance, the CARD of caspase-2 interacts with the CARD present in the adaptor RAIDD. ${ }^{18,19}$ RAIDD also contains a DD in its carboxyl terminal region, which interacts with a DD present in RIP, a serine/threonine kinase that is recruited to tumor necrosis factor receptor 1 (TNFR1) and is required for the TNF-induced activation of NF- $\kappa$ B. ${ }^{18-20}$ The CARD domains in CED-3 and caspase-9 bind CARDs in CED-4 and Apaf-1, respectively. ${ }^{21-23}$ As is the case of FADD/caspase-8, CED-4/CED-3 and Apaf-1/ caspase- 9 interactions are required for caspase activa-

Table 1 Two classes of caspases are found in metazoans

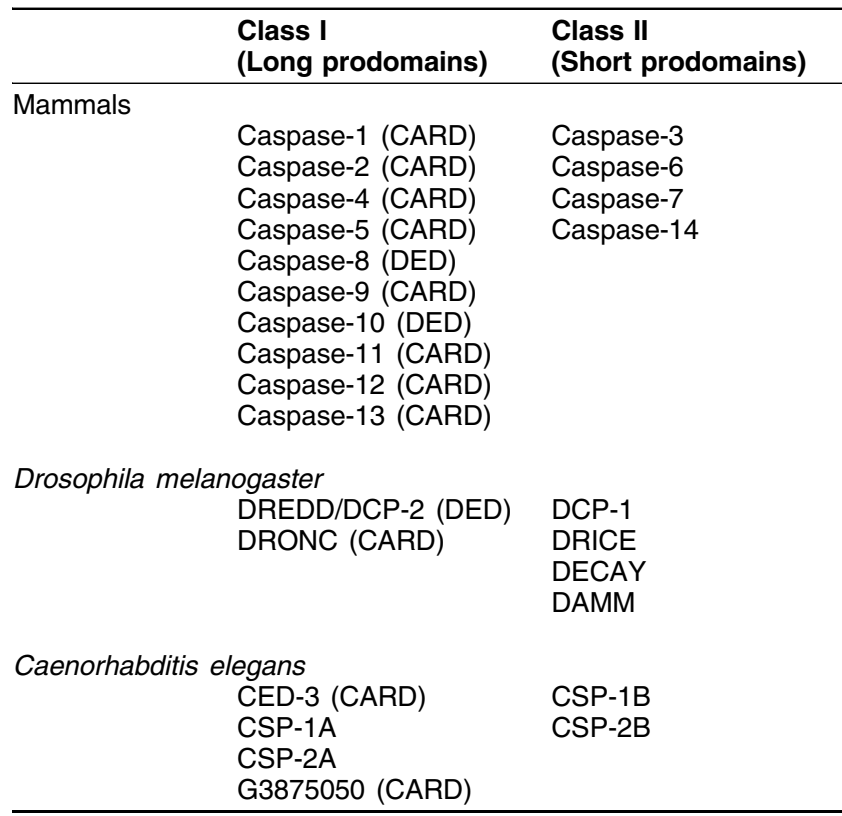

Most class I caspases either contain a CARD or a DED in their prodomain region. For CED-3 and mammalian caspases 1-13 see recent review articles and their references. ${ }^{2-4,11}$ For other caspases, the references are as follows: caspase-14:73 DREDD/DCP-2:74,75 DCP-1:76 DRICE:77 DRONC; ${ }^{17}$ DECAY; 78 CSP-1A, CASP-1B, CSP-2A, CSP-2B. ${ }^{79}$ G3875050 is an unpublished entry in the $C$. elegans sequence database. DAMM is a new Drosophila caspase currently being characterised (N Harvey and S Kumar, unpublished)

Table 2 CARDs and DEDs mediate interactions between procaspases and their respective adaptors

\begin{tabular}{lll}
\hline Adaptor & Caspase & $\begin{array}{l}\text { Domains mediating } \\
\text { interaction }\end{array}$ \\
\hline CED-4 & CED-3 & CARD \\
DARK (?) & DREDD & $(?)$ \\
DARK & DRONC & CARD (?) \\
CARDIAK (?) & caspase-1 & CARD \\
RAIDD/CRADD & caspase-2 & CARD \\
FADD & caspase-8 & DED \\
Apaf-1 & caspase-9 & CARD \\
FADD & caspase-10 & DED \\
\hline
\end{tabular}

tion. $^{21,22}$ However, it is not clear whether interaction between RAIDD and caspase- 2 is necessary for caspase activation.

\section{Domains of death}

From the studies described above, it is clear that homotypic interactions between molecules of the death effector machinery are mediated via DDs, DEDs and CARDs. ${ }^{9,10}$ These interactions are crucial for apoptotic signaling and ensuing caspase activation. The primary function of procaspase/adaptor interactions seems to be to bring several procaspase molecules in close proximity, allowing them to autoproteolytically process themselves. Among the three types of domains mediating interactions between molecules of the death effector apparatus, DDs are commonly found in upstream components of the apoptotic pathways, such as death receptors (e.g. CD95, TNFR1, DR3, DR4 and DR5) and the adaptor molecules that are recruited to these receptors (e.g. FADD, TRADD and RIP). ${ }^{14}$ On the other hand, DEDs and CARDs are generally responsible for recruiting class I caspase precursors to death effector complexes through

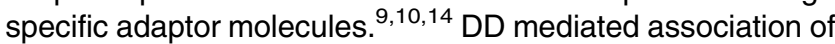
receptors and adaptors usually occurs as a consequence of ligand-dependent activation of death receptors. NMR structures of the CD95 DD, FADD DED and RAIDD CARD have been determined during the past 3 years. ${ }^{24-26}$ All three domains share a very similar structure consisting of six or seven antiparallel amphipathic $\alpha$-helices. There are, however, significant differences between the three domains. For example, mutations of residues in the Fas DD which inhibit protein-protein interactions, have no effect on the FADD DED, and a hydrophobic region in the FADD DED crucial for binding to the caspase- 8 DED is absent in the Fas DD. ${ }^{24,25}$ Furthermore residues required for apoptotic activity in DEDs and DDs are not conserved in CARDs, indicating that the three domains may use a different set of residues to define their binding specificity and function. Electrostatic interactions are predicted to mediate homotypic interactions in DDs and CARDs, whereas the interactions between DEDs are thought to involve hydrophobic regions. ${ }^{24-26}$ Additionally, the orientation of two helices in the RAIDD CARD is significantly different from the corresponding helices in the Fas DD. ${ }^{26}$ The surface of the RAIDD CARD contains a basic region formed by helices 1,3 and 4 , and an acidic region formed by helices 2, 5 and 6. Modeling of the caspase-2 CARD shows a similar structure and surface polarity, suggesting that the interaction between RAIDD and caspase- 2 is mediated by electrostatic bonding between the two CARDs. Although similar protein-protein interactions were suggested, by structural predictions, to occur between the CARDs of Apaf1 and caspase-9, and CED-4 and CED-3, ${ }^{26}$ recent data suggest that there are significant differences from the RAIDD/ caspase- 2 scenario in the way caspase- 9 and Apaf- 1 interact with each other. ${ }^{27}$ Crystal structure of Apaf-1 CARD by itself and in complex with the prodomain of caspase- 9 gives some interesting insights into the interaction between caspase- 9 and Apaf-1. Unlike RAIDD CARD which comprises six $\alpha$ helices, CARDs in Apaf-1 and caspase- 9 consist of seven helices. Two of these helices $(\mathrm{H} 2$ and $\mathrm{H} 3)$ in Apaf-1 form a 
convex acidic surface that is directly involved in recognition and interaction with the basic and concave surface of caspase-9 CARD formed by helices $\mathrm{H} 1 \mathrm{a}, \mathrm{H} 1 \mathrm{~b}$ and $\mathrm{H} 4{ }^{27}$ Although electrostatic interactions favour initial apposition of the two proteins, since high ionic strength does not disrupt the binding, the interactions at the interface are likely to be hydrophobic. ${ }^{27}$ Computer modeling predicts similar interactions at the interface between CED-3 and CED-4 proteins. ${ }^{27}$

\section{Oligomerisation mediates caspase activation}

Although the concept of caspase activation by oligomerisation is not new, it has received much deserved attention only during the past 2 years. It seems that in most cases an aggregation of procaspase molecules is sufficient to mediate their activation. ${ }^{9}$ Although oligomerisation of caspases is mediated by the DEDs and CARDs present in their prodomain regions, often assisted by specific adaptors, forced oligomerisation of class II caspases can also result in caspase activation. ${ }^{28,29}$ This suggests that all caspases, irrespective of whether they have a CARD/DED or not, carry some intrinsic protease activity.

The first study implying oligomerisation in caspase activation was published over 4 years ago when $\mathrm{Gu}$ et al. $^{30}$ showed that oligomerisation of precursor molecules was required for the activation of caspase-1. Subsequent studies using yeast-two hybrid assays demonstrated that procaspase-1 can homodimerise in Saccharomyces cerevisiae, and that homodimerisation is mediated via the prodomain. ${ }^{31}$ Similar results were obtained with caspase$2 .^{32}$ The caspase-2 prodomain is required for homodimerisation of the precursor, and homodimerisation occurs prior to caspase processing. Prodomain-less caspase-2 does not undergo significant processing into subunits when expressed in $S$. cerevisiae suggesting that the prodomain is required for processing of the precursor. ${ }^{32}$ From these studies it became apparent that the primary function of prodomains, at least in caspase-1 and caspase-2, is to mediate procaspase dimerisation and that dimerisation of the precursor is necessary and sufficient for caspase activation.

A number of groups used forced oligomerisation strategies to show that procaspase oligomerisation can indeed induce caspase activation. Both class I caspases, such as CED-3, caspase-8 and caspase-9, and class II caspases, such as caspase-3, are able to undergo proteolytic processing when induced to oligomerise by artificial means. ${ }^{28,33-35}$ In another study, fusion of the caspase-2 prodomain to procaspase-3, a class II caspase unable to autoprocess, was found to be sufficient to mediate autocatalytic activation of the chimeric molecule in transfected cells. ${ }^{29}$ The activation of chimeric caspase-3 was shown to occur as a result of dimerisation via the caspase-2 prodomain. ${ }^{29}$

On the basis of these studies the events leading to the activation of class I caspases can be envisaged to involve the following steps: first, conformational changes due to upstream signalling events result in the recruitment of adaptor molecules to a death complex; second, this is followed by further changes that promote the recruitment of caspases through specific domains; and finally, close proximity of procaspase molecules allows inter- or intramolecular catalysis and activation of the zymogen. The exact mechanism of processing of procaspases is not fully understood, but the fact that procaspase molecules in which the catalytic cysteine residue had been mutated fail to undergo processing in transfecting cells, suggests that autocatalytic activity of caspase zymogens is essential for their processing. ${ }^{1-4,9}$ Indeed, the caspase- 8 zymogen has been shown to possess low levels of enzymatic activity, ${ }^{34}$ and other procaspases are predicted to contain low level protease activity. ${ }^{9}$ This activity may become exposed by proximity-induced dimerisation of precursors allowing refolding and generating a structure similar to that of an active enzyme.

Although, the basic mechanism of activation by oligomerisation appears to be similar for most caspases, there are significant differences between the class I caspases studies so far. Some of the observations related to individual caspases are summarised below.

\section{Caspase-1 and caspase-2}

When overexpressed in mammalian cells caspase-1 and caspase-2 precursors are potent inducers of apoptosis. ${ }^{36-38}$ Initially these observations were somewhat puzzling given that cells can normally tolerate moderate levels of caspase zymogens. One of the predictions was that procaspases normally remain bound to intracellular inhibitors which prevent inappropriate activation. ${ }^{1,2}$ Overexpression in transfected cells may compete out the cellular inhibitor and free precursor molecules are then able to autoactivate in a concentration dependent manner. ${ }^{1,2}$ The potent cell killing activity of these caspases can now, at least in part, be ascribed to the ability of these caspases to form CARDdependent oligomers very efficiently. In addition to the fact that prodomains from both caspase-1 and caspase-2 can dimerise efficiently in yeast two-hybrid assays, ${ }^{31,32}$ upon transfection in mammalian cells, procaspase- 2 and the prodomain of caspase-2 form elaborate filamentous and dot-like higher order structures which can be visualised using GFP-fused proteins. ${ }^{39}$ The formation of these higher order structures is dependent on the caspase-2 CARD. The CARD in caspase-1 precursor interacts with the CARD in CARDIAK/RIP2/RICK, a RIP-like serine/threonine kinase. ${ }^{40-42}$ CARDIAK also associates with TNFR-associated factors TRAF-1 and TRAF- 2 and induces NF- $\kappa$ B activation. ${ }^{40-42}$ Preliminary data suggest that the interaction of CARDIAK with procaspase-1 correlates with the processing of the caspase-1 zymogen, pointing to a potential role for CARDIAK in caspase activation. ${ }^{40}$ Procaspase-2 can efficiently bind the adaptor molecule RAIDD in a CARD-dependent manner, ${ }^{18,19}$ although it is not entirely clear whether this interaction is required for caspase-2 activation. One possibility is that the function of the adaptors CARDIAK and RAIDD is simply to recruit procaspase-1 and -2 to a death signalling complex. Alternatively, these adaptors may be required for stabilizing the caspase oligomers prior to caspase activation. 


\section{Caspase-8, caspase-10 and DREDD}

As mentioned above, caspase- 8 and -10 contain two DEDs in their prodomain region. Most of the studies have focused on caspase-8 which plays a non-redundant role in apoptosis mediated by a number of death receptors. ${ }^{43}$ Although the mechanism of caspase-10 processing has not been studied, given the structural similarities between caspase- 8 and caspase-10, it is likely that caspase-10 is activated by a mechanism similar to that of caspase-8. One of the two DEDs in caspase- 8 interacts with the DED in FADD, recruiting the procaspase to the activated death receptor Fas, ${ }^{15}$ whereas the other DED may be required for homodimerisation or stabilisation of the complex. Similar to caspase-2 CARD, when overexpressed, the DEDs of FADD and caspase-8 form intracellular filaments, termed death effector filaments (DEFs), presumably due to oligomerisation. ${ }^{44}$ DEFs are capable of recruiting and activating procaspases. ${ }^{44}$ Formation of DEFs is not simply due to the overexpression of DEDs, as generation of endogenous DEFs containing FADD and caspase-8 has been seen in Jurkat and CEM cells treated with cycloheximide. ${ }^{45}$ Thus DED-mediated oligomerisation appears to be critical for the activation of DED-containing caspases.

A new molecule, termed FLASH, has recently been cloned and implicated in caspase-8 activation. ${ }^{46}$ FLASH contains a DED-like domain which can interact with the DEDs in both caspase-8 and FADD. In addition, FLASH has a oligomerisation motif which is distantly related to oligomerisation domains in CED-4 and Apaf-1 (see below). Although FLASH is recruited to activated CD95 death signalling complex, the exact mechanism by which FLASH mediates caspase- 8 activation is not understood.

Little is currently known about the activation of DEDcontaining Drosophila caspase DREDD. The structural similarity between DREDD and caspase-8 suggests that DREDD may be involved in a pathway similar to that mediated by mammalian death receptors, but no evidence for this is available as yet. DARK, the recently discovered Drosophila homologue of CED-4/Apaf-1, has been shown to interact with DREDD. ${ }^{47}$ This is somewhat surprising given that DARK does not contain a DED, and the possibility remains that DARK/DREDD interaction is mediated through an additional protein(s). At present, it is also not known whether DARK is required for DREDD activation.

\section{Caspase-9}

The activation of caspase- 9 requires Apaf-1, dATP/ATP and cytochrome $c$, which is released from the mitochondria in cells committed to apoptosis. ${ }^{21}$ Apaf-1 contains multiple WD40 motifs in the carboxyl terminal region which appears to act as a negative regulatory domain by preventing Apaf-1 oligomerisation and Apaf-1/caspase-9 interaction. ${ }^{48-52}$ The deletion of the Apaf-1 WD-40 repeats makes Apaf-1 constitutively active and capable of processing procaspase9 independent of cytochrome $c$ and dATP. ${ }^{48,49}$ Thus, binding of cytochrome $c$ and dATP to Apaf-1 presumably causes conformational changes in the Apaf-1 molecule which exposes the amino terminal CARD, enabling it to interact with the CARD domain in procaspase-9.9 Apaf-1 also contains a region with homology to the $C$. elegans death protein CED-4. ${ }^{21}$ This region is believed to be required for Apaf-1 oligomerisation. ${ }^{48}$ Adaptor oligomerisation through a CED-4-like domain and recruitment of caspase-9 through a CARD motif mediates autocatalytic processing of caspase-9. In reconstitution studies using purified components it was found that Apaf- 1 binds and hydrolyses ATP and dATP. 50,53 This is followed by binding of Apaf- 1 and cytochrome $c$ which promotes multimerisation of Apaf-1 molecules. The multimeric Apaf-1 complex then recruits and activates procaspase-9. ${ }^{53}$ Unlike caspase-1 and caspase-2, caspase-9 appears to have a strict requirement for an adaptor for its activation. Consistent with this prediction, the CARD in caspase- 9 does not homodimerise in yeast (PA Colussi and $S$ Kumar, unpublished data) and does not form higher order structures in a manner analogous to the caspase-2 CARD. ${ }^{39}$ Thus, despite some structural similarities, the CARD in caspase- 9 is functionally distinct from CARDs in caspase- 1 and caspase-2.

In addition to Apaf-1, another similar protein called CARD4/Nod1, has been shown to interact with and activate caspase-9. ${ }^{54,55}$ CARD4/Nod1 contains a CARD, a nucleotide binding sequence and a carboxyl-terminal leucine-rich region. This protein also induces NF- $\kappa \mathrm{B},{ }^{54,55}$ and as yet it is unclear whether CARD4/Nod1 is essential for caspase- 9 activation.

A recent paper reports that procaspase- 9 processing is not required for its activation. ${ }^{56}$ The recombinant procaspase- 9 containing mutations that abolish processing sites of the zymogen was able to activate downstream caspases, but only in the presence of cytosolic factors. The overexpression of this processing-deficient procaspase-9 protein induces apoptosis in transfected cells. These results suggest that caspase- 9 zymogen does not require proteolytic processing, but instead, requires cytosolic factors for expression of its activity. ${ }^{56}$ As rapid processing of caspase-9 is seen in response to many apoptotic signals, the physiological significance of activation without processing is as yet unclear. Perhaps, binding to a cytosolic factor such as Apaf-1 generates an unusually active caspase- 9 conformation prior to processing.

\section{CED-3 and DRONC}

The mechanism of CED-3 activation is similar to caspase-9, as both these caspases require functionally similar adaptors. CED-4, the adaptor for CED-3, however, does not require binding to cytochrome $c$ prior to its interaction with CED-3, and lacks the WD40 repeats present in Apaf-1. ${ }^{21,22}$ CED-4 interacts with CED-3 and this interaction is necessary for CED-3 activation. CED-4 has been shown to oligomerise both in vitro and in vivo, and mutations that abolish CED-4 oligomerisation, inactivate its ability to activate CED-3. ${ }^{57}$ Thus, it seems that analogous to caspase-9, CED-3 oligomerisation can only occur in the presence of its adaptor CED-4. The CARD present in CED-3 is thus unable to homodimerise. Instead, the CED-3 CARD is necessary for its interaction with CED-4.

As yet there is little information available about the mechanism of activation of the CARD-containing Drosphila 
caspase DRONC. However, some recent data suggest that this caspase may be similar to CED-3 and caspase- 9 in the way it is activated. For instance, unlike caspase- 1 and caspase-2 CARDs, DRONC CARD does not homodimerise in $S$. cerevisiae, suggesting that it may require a CED-4/ Apaf-1-like adaptor molecule for its proximity-induced activation (PA Colussi and $S$ Kumar, unpublished data). Preliminary data indicates that DRONC interacts with DARK, ${ }^{47}$ however, more experiments are required to test whether DARK is indeed the adaptor protein required for DRONC activation.

\section{Regulation of procaspase activation}

Being a new area of investigation, regulation of caspase activation is relatively poorly understood, however some interesting insights are beginning to appear. It is now becoming increasingly clear that like any other critical cellular mechanism, the activation of class I caspases is a tightly regulated and complex process. Many recent studies indicate that the control of caspase activation is achieved both at the level of caspase recruitment and procaspase oligomerisation. The long known Bcl-2 family members which lie upstream of caspase cascades, appear to regulate events just prior to caspase/adaptor interactions. ${ }^{58}$ For instance the $C$. elegans antiapoptotic Bcl-2-like protein CED-9 blocks death by complexing with CED-4 and attenuating its ability to promote CED-3 activation. ${ }^{57}$ The binding of CED-9 to CED-4 blocks oligomerisation of CED-4 molecules suggesting that CED-9 inhibits apoptosis primarily by modulating oligomerisation mediated caspase activation. ${ }^{57}$ Similar interactions apparently occur in mammalian cells involving Apaf-1, the mammalian death protease caspase- 9 , and $\mathrm{Bcl}-\mathrm{X}_{\mathrm{L}}$, an antiapoptotic member of the Bcl-2 family. ${ }^{59} \mathrm{Bcl}-2$ also functions to block release of cytochrome $c$ from mitochondria, thereby suppressing Apaf-1-mediated activation of caspase-9. ${ }^{60}$ On the other hand, Bid, the proapoptotic member of Bcl-2 family carrying a single $\mathrm{Bcl}-2$ homology domain (BH3), appears to promote release of cytochrome $c .{ }^{61,62}$ Another anti-apoptotic member of the Bcl-2 family, Boo, interacts with Apaf- 1 and forms a multimeric protein complex with Apaf-1 and caspase9. ${ }^{63}$ Bak and Bik, two pro-apoptotic Bcl-2 family members, disrupt the association of Boo and Apaf-1. In an analogous fashion, the $C$. elegans protein EGL-1 disrupts the interaction between CED-9 and CED-4 and promotes CED-3 activation. ${ }^{64}$ Since there are a large number of pro- and antiapoptotic Bcl-2-like proteins, it seems likely that they act in a cell and signal specific manner by modulating events that regulate oligomerisation of certain caspases.

Catalytically inactive molecules which mimic caspases also appear to play a key role in the regulation of caspase activation. An endogenous alternatively spliced caspase-9 mRNA encodes a protein that lacks most of the large subunit but has an intact CARD. ${ }^{65,66}$ The expression of this inactive caspase-9 like molecule inhibits multiple forms of apoptosis, including that mediated by the death receptors, and appears to act by interfering with the formation of a functional Apaf-1/caspase-9 complex. Several other CARD and DED containing regulators of caspase activation have recently been identified, based mostly on homology searching of sequence databases. Most of these appear to function by interfering with caspase recruitment and/or oligomerisation. Caspase-8-like molecules such as viral and cellular FLIPs, which contain DEDs but lack catalytic function inhibit caspase- 8 activation by blocking caspase- 8 recruitment to $\mathrm{FADD} .^{3,11} \mathrm{ARC}$, a molecule that contains a CARD, interacts with caspase-2, -8 and CED-3, but not with caspase-1, -3 , or $-9 .{ }^{67}$ ARC inhibits apoptosis induced by CED-3 and caspase-8 but not that induced by caspase9 overexpression. The interaction of ARC with caspase-8, which seems to involve DEDs of caspase-8, is somewhat surprising given that caspase-8 does not contain a CARD. The expression of ARC is restricted to skeletal muscle and cardiac tissues, and it has been suggested to be a tissue specific inhibitor of caspase activation. ${ }^{67}$

Bcl10, a gene encoding a CARD containing molecule, lies near the recurrent breakpoint in MALT B cell lymphoma and is mutated in a number of other tumors. ${ }^{68} \mathrm{Bcl} 10$ is a cellular homologue of the equine herpesvirus-2 E10 gene. Wild-type Bcl10 suppresses transformation, whereas mutant forms display gain-of-function transforming activity. Overexpression of the $\mathrm{Bcl} 10$ protein, also called $\mathrm{mE} 10,{ }^{69}$ CIPER $^{70}$ and CARMEN, ${ }^{71}$ induces apoptosis which is dependent on CARD-mediated oligomerisation of the protein. Interestingly, while the CARD of mE10 is required for oligomerisation, the carboxyl terminus interacts with procaspase- 9 and promotes its processing. ${ }^{69}$ Expression of CIPER also induces NF- $\kappa$ B activation, ${ }^{70,71}$ apparently in a NIK-dependent manner. ${ }^{70}$

Another form of regulation of caspase activation has recently come to light when it was shown that Akt, a serine/ threonine kinase involved in some cell survival pathways, and p21-Ras, an activator of Akt, induce phosphorylation of procaspase-9. ${ }^{72}$ In cytosolic extracts prepared from cells expressing either active Ras or Akt, the cytochrome $c$ dependent activation of caspase- 9 is abrogated, suggesting that phosphorylation of procaspase- 9 inhibits its processing and activation. Although it is not entirely clear how phosphorylation inhibits caspase- 9 processing, it is suggested that phosphorylation may effect dimerisation via an allosteric mechanism. ${ }^{72}$

\section{Conclusions}

From the many studies summarised above it is clear that homotypic protein-protein interactions through specific domains are key to the regulation of caspase activation. In a simple scenario, one can envisage that specific death signals lead to conformational changes in adaptors and caspases allowing them to interact with each, which in turn result in proximity induced refolding and autocatalysis of caspases. While caspases such as CED-3 and caspase-9 require CARD-mediated interactions with their respective adaptors, the function of adaptors in the activation of caspase-1 and caspase-2 is less clear. Thus, it appears that CARDs in various caspases and adaptors function differently. While caspase-2 and RAIDD CARDs can homodimerise and heterodimerise, the CARDs in CED-3, CED-4, Apaf-1 and caspase- 9 seem to lack the ability to form homodimers. DED containing caspases, such as caspase-8, and its adaptor 
FADD seem to have the ability to both homodimerise and heterodimerise. These apparently subtle differences between the ability of caspases to oligomerise may have significant physiological implications. For example, since caspases such as caspase- 2 and caspase- 8 can be activated by self aggregation, the cellular concentration of these caspases may be crucial in sensitising cells to apoptosis. On the other hand, since caspase- 9 and CED-3 have an absolute requirement for an adaptor molecule to mediate their oligomerisation, both the caspase and adaptor concentrations and accessibility of these molecules to contact each other will determine the vulnerability of a cell to apoptosis.

Numerous molecules that appear to regulate the activation of caspases have come to light in recent years. In apoptotic pathways that involve Apaf-1/caspase-9, the Bcl-2 family of proteins appear to play a key role in controlling caspase activation. Many other molecules contain protein-protein interaction domains that resemble those found in class I caspases and their adaptors. These molecules also appear to primarily regulate caspase activation by directly or indirectly modulating the ability of procaspases to oligomerise. One area of caspase activation which is still not well elucidated is the exact mechanism by which a caspase zymogen is activated following oligomerisation. Since the three-dimensional structure of procaspases is not known, it is not clear how caspase precursor molecules can fold into an active enzyme. No doubt there is much more to learn about caspase activation and given the complexity of most biological processes, there may still be surprises in the offing.

\section{Acknowledgements}

I thank Paul Colussi for discussions and useful comments on the manuscript. Work in my laboratory is supported by the Wellcome Trust and the National Health and Medical Research Council of Australia.

\section{References}

1. Kumar S (1995) ICE-like proteases in apoptosis. Trends Biochem. Sci. 20: 198 202

2. Kumar S and Lavin MF (1996) The ICE family of cysteine proteases as eefectors of cell death. Cell Death Differ. 3: 255-267

3. Nicholson DW and Thornberry NA (1997) Caspases: killer proteases. Trends Biochem. Sci. 22: 299-306

4. Kumar S (1999) Regulation of caspase activation in apoptosis: Implications in pathogenesis and treatment of disease. Clin. Expt. Pharmacol. Physiol. 26 $295-303$

5. Wilson KP, Black J-AF, Thomson JA, Kim EE, Griffith JP, Navia MA, Murcko MA, Chambers SP, Aldape RA, Raybuck SA and Livingston DJ (1994) Structure and mechanism of interleukin-1 $\beta$ converting enzyme. Nature 370: 270-275

6. Walker NPC, Talanian RV, Brady KD, Dang LC, Bump NJ, Ferenz CR, Franklin S, Ghayur T, Hackett CR, Hammill LD, Herzog L, Hugunin M, Houy W, Mankovich JA, McGuiness L, Orlewicz E, Paskind M, Pratt CA, Reis P, Summani A, Terranova M, Welch JP, Xiong L, Möller A, Tracey DE, Kamen R and Wong WW (1994) Crystal structure of the cysteine protease interleukin-1 $\beta$ converting enzyme: a (p20/p10) 2 homodimer. Cell 78: 343-352

7. Rotonda J, Nicholson DW, Fazil KM, Gallant M, Gareau Y, Labelle M, Peterson EP, Rasper DM, Ruel R, Vaillancourt JP, Thornberry NA and Becker JW (1996) The three-dimensional structure of apopain/CPP32, a key mediator of apoptosis. Nat. Struct. Biol. 3: 619-625
8. Mittl PR, Di Marco S, Krebs JF, Bai X, Karanewsky DS, Priestle JP, Tomaselli KJ and Grutter MG (1997) Structure of recombinant human CPP32 in complex with the tetrapeptide acetyl-Asp-Val-Ala-Asp fluoromethyl ketone. J. Biol. Chem. 272: $6539-6547$

9. Kumar S and Colussi PA (1999) Prodomains-adaptors-oligomerization: the pursuit of caspase activation in apoptosis. Trends Biochem. Sci. 24: 1-4

10. Aravind L, Dixit VM and Koonin EV (1999) The domains of death: evolution of apoptosis machinery. Trends Biochem. Sci. 24: 47-53

11. Rathmell JC and Thompson CB (1999) The central effectors of cell death in the immune system. Annu. Rev. Immunol. 17: 781-828

12. Boldin MP, Goncharov TM, Goltsev YV and Wallach D (1996) Involvement of $\mathrm{MACH}$, a novel Mort1/FADD-interacting protease, in Fas/APO1- and TNF receptor-induced cell death. Cell. 85: 803-815

13. Muzio M, Chinnaiyan AM, Kischkel FC, O'Rourke K, Shevchenko A, Scaffidi C Bretz JD, Zhang M, Ni J, Gentz R, Mann N, Krammer PH, Peter ME and Dixit VM (1996) FLICE, a novel FADD-homologous ICE/CED-3-like protease, is recruited to the CD95 (Fas/APO-1) death-inducing signaling complex. Cell 85: 817-827

14. Ashkenazi A and Dixit VM (1998) Death receptors: signaling and modulation. Science 281: $305-308$

15. Medema JP, Scaffidi C, Kischkel FC, Shevchenko A, Mann M, Krammer PH and Peter ME (1997) FLICE is activated by association with the CD95 death-inducing signalling complex (DISC). EMBO J. 16: 2794-2804

16. Hofman K, Bucher P and Tschopp J (1997) The CARD domain: a new apoptotic signalling motif. Trends Biochem. Sci. 22: 155-156

17. Dorstyn L, Colussi PA, Quinn LM, Richardson Hand Kumar S (1999) DRONC, an ecdysoneinducible Drosophila caspase. Proc. Natl. Acad. Sci. USA 96: 4307 4312

18. Ahmad M, Srinivasula SM, Wang L, Talanian RV, Litwak G, Fernandes-Alnemri T and Alnemri ES (1997) CRADD, a novel human apoptotic adaptor molecule for caspase-2, and FasL/tumor necrosis factor receptor-interacting protein RIP. Cancer Res. 57: 615-619

19. Duan H and Dixit VM (1997) RAIDD is a new 'death' adaptor molecule. Nature 385: $86-89$

20. Kelliher MA, Grimm S, Ishida Y, Kuo F, Stanger BZ and Leder P (1998) The death domain kinase RIP mediates the TNF-induced NF-kappaB signal. Immunity. 8: $297-303$

21. Li P, Nijhawan D, Budihardjo I, Srinivasula SM, Ahmad M, Alnemri ES and Wang $X$ (1997) Cytochrome $c$ and dATP-dependent formation of Apaf-1/caspase-9 complex initiates an apoptotic protease cascade. Cell 91: 479-489

22. Chinnaiyan AM, Chaudhary D, O'Rourke K, Koonin EV and Dixit VM (1997) Role of CED-4 in the activation of CED-3. Nature 388: 728-729

23. Chaudhary D, O'Rourke K, Chinnaiyan AM and Dixit VM (1998) The death inhibitory molecules CED-9 and CED-4L use a common mechanism to inhibit the CED-3 death protease. J. Biol. Chem. 273: 17708-17712

24. Huang B, Eberstadt M, Olejniczak ET, Meadows RP and Fesik SW (1996) NMR structure and mutagenesis of the Fas (APO-1/jCD95) death domain. Nature 384: $638-641$

25. Eberstadt M, Huang B, Chen Z, Meadows RP, Ng S-I, Zheng L, Lenardo MJ and Fesik SW (1998) NMR structure and mutagenesis of the FADD (Mort1) deatheffector domain. Nature 392: $941-945$

26. Chou JJ, Matsuo H, Duan H and Wagner G (1998) Solution structure of the RAIDD CARD and model for CARD/CARD interaction in caspase-2 and caspase-9 recruitment. Cell. 94: 171-180

27. Qin H, Srinivasula SM, Wu G, Fernandes-Alnemri T, AlnemriES and Shi Y (1999) Structural basis of procaspase- 9 recruitment by the apoptotic proteaseactivating factor 1. Nature 399: $549-557$

28. MacCorkle RA, Freeman KW and Spencer DM (1998) Synthetic activation of caspases: artificial death switches. Proc. Natl. Acad. Sci. USA. 95: 3655-3660

29. Colussi PA, Harvey NL, Shearwin-Whyatt LM and Kumar S (1998) Conversion of procaspase-3 to an autoactivating caspase by fusion to the caspase-2 prodomain. J. Biol. Chem. 273: 26566-26570

30. Gu Y, Wu J, Faucheu C, Lalanne JL, Diu A, Livingston DJ and Su MS (1995) Interleukin-1 beta converting enzyme requires oligomerization for activity of processed forms in vivo. EMBO J. 14: 1923-1931

31. Van Criekinge W, Beyaert R, Van de Craen M, Vandenabeele P, Schotte P, De Valck D and Friers W (1996) Functional characterization of the prodomain of interleukin-1beta-converting enzyme. J. Biol. Chem. 271: 27245-27248

32. Butt AJ, Harvey NL, Parasivam G and Kumar S (1998) Dimerization and autoprocessing of the Nedd2(caspase-2) precursor requires both the prodomain and the carboxyl-terminal regions. J. Biol. Chem. 273: 6763-6768 
33. Martin DA, Siegel RM, Zheng L and Lenardo MJ (1998) Membrane oligomerization and release activates the caspase 8 (FLICE/MACH $\alpha 1)$ death signal. J. Biol. Chem. 273: 4345-4349

34. Muzio M, Stockwell BR, Stennicke HR, Salvesen GS and Dixit VM (1998) An induced proximity model for caspase-8 activation. J. Biol. Chem. 273: 29622930

35. Yang X, Chang HY and Baltimore D (1998) Autoproteolytic activation of procaspases by oligomerization. Mol. Cell 1:319-325

36. Miura M, Zhu H, Rotello R, Hartwieg EA and Yuan J (1993) Induction of apoptosis in fibroblasts by IL- 1 beta-converting enzyme, a mammalian homolog of the $C$. elegans cell death gene ced-3. Cell 75: 653-660

37. Kumar S, Kinoshita M, Noda M, Copeland NG and Jenkins NA (1994) Induction of apoptosis by the mouse Nedd2 gene, which encodes a protein similar to the product of the Caenorhabditis eleganscell death gene ced-3 and the mammalian IL-1 $\beta$-converting enzyme. Genes Dev. 8: 1613-1626

38. Dorstyn $L$ and Kumar S (1997) Differential inhibitory effects of CrmA, P35, IAP and three mammalian IAP homologues on apoptosis in NIH3T3 cells following various death stimuli. Cell Death Differ. 4: 570-579

39. Colussi PA, Harvey NL and Kumar S (1998) Prodomain-dependent nuclear localization of the caspase-2(Nedd2) precursor. A novel function for a caspase prodomain. J. Biol. Chem. 273: 24535-24542

40. Thome M, Hofmann K, Burns K, Martinon F, Bodmer JL, Mattmann C and Tschopp J (1998) Identification of CARDIAK, a RIP-like kinase that associates with caspase-1. Curr. Biol. 8: 885-888

41. McCarthy JV, Ni J and Dixit VM (1998) RIP2 is a novel NF-kappaB-activating and cell death-inducing kinase. J. Biol. Chem. 273: 16968-16975

42. Inohara N, del Peso L, Koseki T, Chen S and Núñez G (1998) RICK, a nove protein kinase containing a caspase recruitment domain, interacts with CLARP and regulates CD95-mediated apoptosis. J. Biol. Chem. 273: 12296-12230

43. Varfolomeev EE, Schuchmann M, Luria V, Chiannikulchai N, Beckman JS, Mett IL, Rebrikov D, Brodianski VM, Kemper OC, Kollet O, Lapidot T, Soffer D, Tama S, Avraham KB, Goncharov T, Holtmann H, Lonai P and Wallach D (1998) Targeted disruption of the mouse Caspase 8 gene ablates cell death induction by the TNF receptors, Fas/Apo1, and DR3 and is lethal prenatally. Immunity 9:267 276

44. Siegel RM, Martin DA, Zheng L, Ng SY, Bertin J, Cohen J and Lenardo MJ (1998) Death-effectorfilaments: Novel cytoplasmic structures that recruit caspases and trigger apoptosis. J. Cell. Biol. 141: 1243-1253

45. Tang D, Lahti JM, Grenet J and Kidd VJ (1999) Cycloheximide-induced T-cell is mediated by a fas-associated death domain-dependent mechanism. J. Biol. Chem. 274: $7245-7252$

46. Imai Y, Kimura T, Murakami A, YajimaN, SakamakiKand Yonehara S(1999) The CED-4-homologous protein FLASH is involved in Fas-mediated activation of caspase-8 during apoptosis. Nature 398: 777-785

47. Rodriguez A, Oliver H, Zou H, Chen P, Wang X and Abrams JM (1999) Dark is a Drosophila homologue of Apaf-1/CED-4 and functions in an evolutionarily conserved death pathway. Nat. Cell Biol. 1: 272-279

48. Srinivasula SM, Ahmed M, Fernandes-Alnemri T and Alnemri ES (1998) Autoactivation of procaspase- 9 by Apaf-1-mediated oligomerization. Mol. Cell 1: 949-957

49. Hu Y, Ding L, Spencer DM and Núñez G (1998) WD-40 repeat region regulates Apaf-1 self-association and procaspase-9 activation. J. Biol. Chem. 273: 33489-33494

50. Hu Y, Benedict MA, Ding L and Núñez G (1999) Role of cytochrome cand dATP/ ATP hydrolysis in Apaf-1-mediated caspase- 9 activation and apoptosis. EMBO J. 18: 3586-3595

51. Adrain C, Slee EA, Harte MT and Martin SJ (1999) Regulation of apoptotic protease activating factor-1 oligomerization and apoptosis by the WD-40 repeat region. J. Biol. Chem. 274: 20855-20860

52. Saleh A, Srinivasula SM, Acharya S, Fishel R and Alnemri ES (1999) Cytochrome $c$ and dATP-mediated oligomerization of Apaf- 1 is a prerequisite for procaspase-9 activation. J. Biol. Chem. 274: 17941-17945

53. Zou H, Li Y, Liu X and Wang X (1999) An APAF-1-cytochrome c multimeric complex is a functional apoptosome that activates procaspase-9. J. Biol. Chem. 274: $11549-11556$

54. Bertin J, Nir WJ, Fischer CM, Tayber OV, Errada PR, Grant JR, Keilty JJ, Gosselin ML, Robison KE, Wong GH, Glucksmann MA and DiStefano PS (1999) Human CARD4 protein is a novel CED-4/Apaf-1 cell death family member that activates NF- $\kappa$ B. J. Biol. Chem. 274: 12955-12958
55. Inohara N, Koseki T, del Peso L, Hu Y, Yee C, Chen S, Carrio R, Merino J, Liu D, Ni J and Núñez G (1999) Nod1, an Apaf-1-like activator of caspase-9 and nuclear factor- $\kappa$ B. J. Biol. Chem. 274: 14560-14567

56. Stennicke HR, Devaraux QL, Humke EW, Reed JC, Dixit VM and Salvesen GS (1999) Caspase-9 can be activated without proteolytic processing. J. Biol. Chem. 274: 8359-8362

57. Yan X, Chang HY and Baltimore D (1998) Essential role of CED-4 oligomerisation in CED-3 activation and apoptosis. Science 281: 1355-1357

58. Adams JM and Cory S (1998) The Bcl-2 protein family: Arbiters of cell survival. Science 281: $1322-1326$

59. Pan G, O'Rourke K and Dixit VM (1998) Caspase-9, Bcl-XL, and Apaf-1, form a ternary complex. J. Biol. Chem. 273: 5841-5845

60. Green DR and Reed JC (1998) Mitochondria and apoptosis. Science 281: $1309-1312$

61. $\mathrm{LiH}, \mathrm{ZhuH}, \mathrm{XuCJ}$ and Yuan J (1998) Cleavage of BID by caspase 8 mediates the mitochondrial damage in the Fas pathway of apoptosis. Cell 94: 491-501

62. LuoX, Budihardjo I, Zou H, Slaughter CandWangX(1998) Bid, a Bcl2 interacting protein, mediates cytochrome $c$ release from mitochondria in response to activation of cell surface death receptors. Cell 94: $481-490$

63. Song Q, Kuang Y, Dixit VM and Vincenz C (1999) Boo, a novel negative regulator of cell death, interacts with Apaf-1. EMBO J. 18: 167-178

64. del Peso L, González VM and Núñez G (1998) Caenorhabditis elegans EGL-1 disrupts the interaction of CED-9 with CED-4 and promotes CED-3 activation. J. Biol. Chem. 273: 33495-33500

65. Seol DW and Billiar TR (1999) A caspase-9 variant missing the catalytic site is an endogenous inhibitor of apoptosis. J. Biol. Chem. 274: 2072-2076

66. Srinivasula SM, Ahmad M, Guo Y, Zhan Y, LazebnikY, Fernandes-Alnemri T and Alnemri ES (1999) Identification of an endogenous dominant-negative short isoform of caspase-9 that can regulate apoptosis. Cancer Res. 59: 999-1002

67. Koseki T, Inohara N, Chen S and Núñez G (1998) ARC, an inhibitor of apoptosis expressed in skeletal muscle and heart that interacts selectively with caspases. Proc. Natl. Acad. Sci. USA 95: 5156-5160

68. Willis TG, Jadayel DM, Du MQ, Peng H, Perry AR, Abdul-Rauf M, Price H, Karan L, Majekodunmi O, Wlodarska I, Pan L, Crook T, Hamoudi R, Isaacson PG and Dyer MJ (1999) Bcl10 is involved in $t(1 ; 14)(p 22 ; q 32)$ of MALT B cell lymphoma and mutated in multiple tumor types. Cell 96: $35-45$

69. Yan M, Lee J, Schilbach S, Goddard A and Dixit V (1999) mE10, a novel caspase recruitment domain-containing proapoptotic molecule. J. Biol. Chem. 274: $10287-10292$

70. Koseki T, Inohara N, Chen S, Carrio R, Merino J, Hottiger MO, Nabel GJ and Núñez G (1999) CIPER, a novel NF kappaB-activating protein containing a caspase recruitment domain with holology to herpesvirus-2 protein E10. J. Biol. Chem. 274: 9955-9961

71. Thome M, Martinon F, Hofmann K, Rubio V, Steiner V, Schneider P, Mattmann C and Tschopp J (1999) Equine herpesvirus-2E10 gene product, but not its cellular homologue, activates NF-kappaB transcription factor and c-Jun N-terminal kinase. J. Biol. Chem. 274: 9962-9968

72. Cardone MH, Roy N, Stennicke HR, Salvesen GS, Franke TF, Stanbridge E, Frisch S and Reed JC (1998) Regulation of cell death protease caspase-9 by phosphorylation. Science 282: 1318-1321

73. Hu S, Snipas SJ, Vincenz C, Salvesen G and Dixit VM (1998) Caspase-14 is a novel developmentally regulated protease. J. Biol. Chem. 273: 29648-29653

74. Chen P, Rodriguez A, Erskine R, Thach T and Abrams JM (1998) Dredd, a novel effector of the apoptosis activators reaper, grim, and hid in Drosophila. Dev. Biol. 201: 202-216

75. Inohara N, Koseki T, Hu Y, Chen S and Núñez G (1997) CLARP, a death effector domain containing protein interacts with caspase-8 and regulates apoptosis. Proc. Natl. Acad. Sci. USA 94: 10717-10722

76. Song Z, McCall K and Steller H (1997) DCP-1 a Drosophila cell death protease essential for development. Science 275: 536-540

77. Fraser AG and Evan GI (1997) Identification of a Drosophila melanogaster ICE/CED-3-related protease, drICE. EMBO J. 16: 2805-2813

78. Dorstyn L, Read SH, Quinn LM, Richardson H and Kumar S (1999) DECAY, a novel Drosophila caspase related to mammalian caspase-3 and caspase-7. J. Biol. Chem. 274: 30778-30783

79. Shaham S (1998) Identification of multiple Caenorhabditis elegans caspases and their potential roles in proteolytic cascades. J. Biol. Chem. 273: 3510935117 